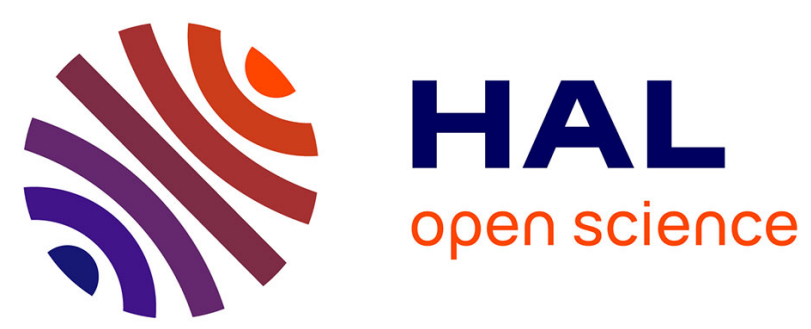

\title{
Real-time Stereo Visual Servoing Control of an Eight-Rotors Rotorcraft
}

\author{
S. Salazar, H. Romero, Jose-Ernesto Gomez-Balderas, R. Lozano
}

\section{To cite this version:}

S. Salazar, H. Romero, Jose-Ernesto Gomez-Balderas, R. Lozano. Real-time Stereo Visual Servoing Control of an Eight-Rotors Rotorcraft. 6th International Conference on Electrical Engineering, Computing Science and Automatic Control. Toluca, Mexique, 2009, pp.1-11. hal-00984480

\section{HAL Id: hal-00984480 \\ https://hal.science/hal-00984480}

Submitted on 28 Apr 2014

HAL is a multi-disciplinary open access archive for the deposit and dissemination of scientific research documents, whether they are published or not. The documents may come from teaching and research institutions in France or abroad, or from public or private research centers.
L'archive ouverte pluridisciplinaire HAL, est destinée au dépôt et à la diffusion de documents scientifiques de niveau recherche, publiés ou non, émanant des établissements d'enseignement et de recherche français ou étrangers, des laboratoires publics ou privés. 


\title{
Real-time Stereo Visual Servoing Control of an UAV having Eight-Rotors
}

\author{
Sergio Salazar, Hugo Romero, Jóse Gómez and Rogelio Lozano
}

\begin{abstract}
This paper presents a visual feedback control of a small UAV using image-based visual servoing with stereo vision. In order to control the orientation and the position of flying robot with respect to an object or target well defined, we propose to use a navigation system based on binocular vision system combined with inertial sensors. This combination of sensors, allows us to get a complete characterization of the state of aerial vehicle. It means, using the stereo vision system we are able to estimate the UAV 3D position, while from the inertial sensors we can obtain the orientation of rotorcraft. Real time experiences are developed to validate the performance of navigation system proposed.
\end{abstract}

Keywords Visual Servoing - Stereo Vision - UAV - Control.

\section{INTRODUCTION}

Small Unmanned Aerial Vehicles (UAV) have became important platforms in several applications both in civilian field and military field where the situational awareness can be drastically augmented if one or more UAVs are deployed for collecting data, surveillance and espionage. This segment of UAVs have many advantages over other segments. The advantages with respect to larger UAVs segment include portability, lower cost, easier handling and operation which can be can performed by a single person. Moreover, they can operate almost inadvertently which makes them ideal for stealth missions. In comparison with the smaller UAVs segment, the small UAVs are more robust to perform outdoor missions under challenging weather conditions. However, the small UAVs have a limited payload, which is an important issue to consider in the choice of sensors to be used in this segment of aerial vehicles. For these reasons, nowadays many research groups are dedicated to developing new UAV configurations, new control laws and new sensing systems to measure and/or estimate the entire aerial vehicles state and to know their surrounding environment.

A standar UAV navigation system combines an Inertial Measurement Unit (IMU) with Global Positioning System (GPS), ultrasonic sensors, laser scanners or radars to estimate the entire state of aerial vehicle [13][17].Nevertheless, the standard techniques based on GPS for navigation are not reliable in urban or hostile environments, because the communication signals with the satellites can easily be blocked or jammed, which implies an erroneous location estimation of the flying machine. Laser scanners and radars are used to estimate the UAV position too, but mainly is applied to avoid obstacles with high accuracy, unfortunately these kind of sensors are expensive

H. Romero, S. Salazar and R. Lozano are with LAFMIAA CINVESTAV, Av. IPN 2508 Col. San Pedro Zacatenco 07360 Mexico, D.F.

sergio.salazar.cruz@gmail.com,

rhugo@uaeh.edu.mx,rlozano@hds.utc.fr

J. Gómez is with HEUDIASyC UTC, Centre de recherches de Royallieu, 60205 Compiegne France. jgomezba@hds.utc.fr

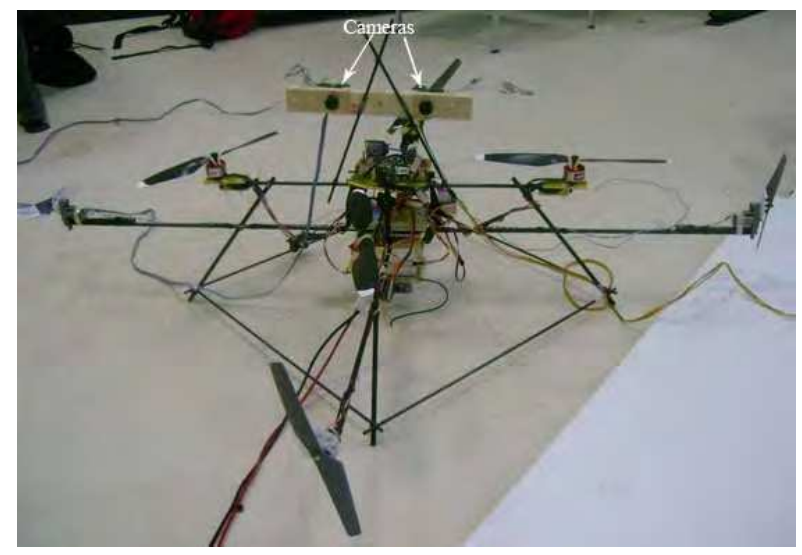

Fig. 1. Eight-rotor rotorcraft whit a stereo vision system home made mounted.

and too heavy to be used in small size flying robots due to their limited payload capacity.

An alternative sensing system is composed by IMU and vision system, it means the UAV is controlled using visual servoing [15][7][21][6]. Visual servoing is the fusion of results from many elemental areas including high-speed image processing, kinematics, dynamics, control theory, and real-time computing. The advantages of combining these two sensor modalities is the complementary characteristics of vision system and inertial sensors. On one hand, the inertial sensors have large measurement uncertainty at slow motion and lower relative uncertainty at high velocities. Inertial sensors can measure high velocities and accelerations. On the other hand, cameras can track features very accurately at low velocities.

Recently some works demonstrates several applications for UAVs using visual systems in [8] a schema to calculate the pose of UAV using two cameras is proposed, applying the unscented Kalman filter (UKF). A vision systems which consists of one off-the-shelf is used in [9] for landing an UAV, this system apply an image processing followed by estimation and control of a camera, other targets such as a landing pad is used in [10], a method for attitude computation catadioptric images for UAV is used in [11] with a catadioptric vision sensor.

The paper presents a stereo vision system to estimate the UAV position, using a control approach for position. The control system decouple the rotational dynamics and translational dynamics of this UAV configuration. It means one or two cameras located in different places taking a photo of the same scene through translating or rotating. A basic problem in stereo vision is to perform $3 \mathrm{D}$ reconstruction of a world scene or object from a pair of images. Stereo vision system is used to compute the altitude and relative displacement of the eight rotor 
rotorcraft with respect to a well know target.

This paper is organized as follows: Section II is devoted to describe the stereo vision theory and tools. The $3 D$ reconstruction method is presented in section III. The platform architecture used in this experience is described in Section IV. The mathematical nonlinear model using the Newton-Euler approach of the multi-rotor mini rotorcraft is presented in Section V. In Section VI the control strategy to stabilize at hover the multi-rotor platform is introduced. Finally, experimental results and conclusions are presented in Section VII and Section VIII respectively.

\section{Stereo Vision}

Visual servo stereo systems typically use one of two cameras in two different configurations: robot mounted, or fixed in the workspace. A stereo vision system must solve mainly two problems. The first problem is known as correspondence, this is to determine which item (points, lines, surfaces, etc.) from the left (right) image corresponds to same item in the right (left) image. The second problem is the reconstruction, it consists in obtain some information about the 3D real world from the stereo vision geometry and matched points from left and right images. Figure 2 represents image-based visual servo structure.

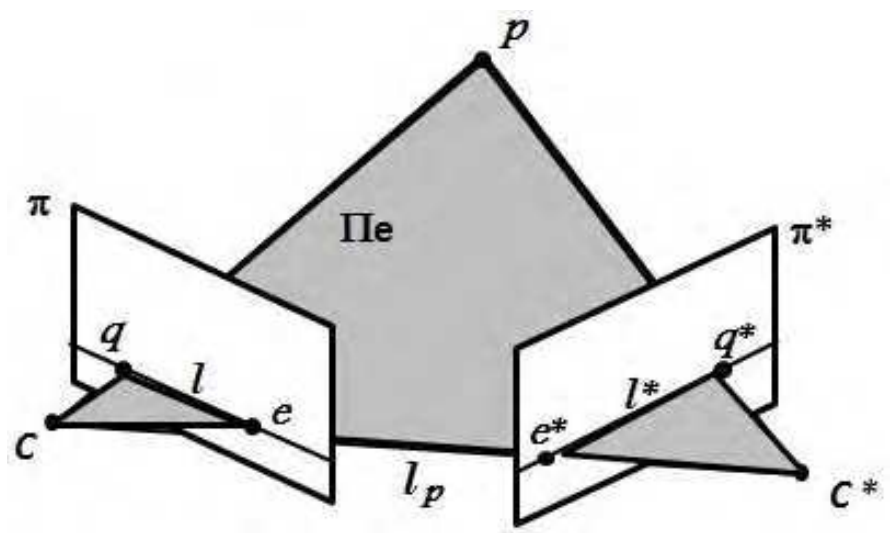

Fig. 2. Basic scheme of epipolar geometry.

Projective and metric relationships in a stereo vision system are based on the epipolar geometry. Under this geometry, the left camera focus $\mathbf{C}$, right camera focus $\mathbf{C}^{*}$ and the real point $p$ form the entity $\Pi_{e}$, which is called the epipolar plane. The intersections of this epipolar plane with the two image planes form the epipolar lines $\mathbf{l}$ and $\mathbf{l}^{*}$ on the left image plane $\pi$ and right image plane $\pi^{*}$ respectively. While the base-line $\mathrm{l}_{b}$ connecting the two centers of projection $\left(\mathbf{C}, \mathbf{C}^{*}\right)$ intersects the image planes at the conjugate points $\mathbf{e}$ and $\mathbf{e}^{*}$ which are called epipoles. Assume that the $3 \mathrm{D}$ point $p$ projects into the left and right image planes as the points $q=(\gamma, \rho, 1)$ and $q^{*}=\left(\gamma^{*}, \rho^{*}, 1\right)$ respectively in homogeneous coordinates. Then, a couple of corresponding points $q \rightarrow q^{*}$ are related as follows

$$
q^{* T} \mathbf{F} q=0
$$

where $q^{* T}$ denotes the transpose of $q^{*}$ and $\mathbf{F}$ is the fundamental matrix which only depends of correlated points $q \rightarrow q^{*}$. It is a $3 \times 3$ homogeneous matrix with rank 2 having 7 degrees of freedom, it means that one of its eigenvalues is equal to zero.

Furthermore, $\mathbf{l}^{*}=\mathbf{F} q$ is the epipolar line onto right image plane, consequently the corresponding point $q^{*} \in \mathbf{l}^{*}$. Similarly $\mathbf{l}=\mathbf{F}^{T} q^{*}$, with $q \in \mathbf{l}$. These relationships are very advantageous from computational cost point of view, because the search of the corresponding point $q^{*}(q)$ is restricted only to epipolar line $\mathrm{l}^{*}(\mathbf{l})$ and not to the whole image $\pi^{*}(\pi)$.

In order to compute the matrix $\mathbf{F}$, let $\mathbf{Q}$ and $\mathbf{Q}^{*}$ be two matrix containing the image point $q_{i}$ and its corresponding image points $q_{i}^{*}$ respectively with $i=1,2, \ldots, n$, then we can set the follow matrix equation

$$
\mathbf{Q}^{* \mathbf{T}} \mathbf{F} \mathbf{Q}=\left[\begin{array}{ccc}
\gamma_{1}^{*} & \rho_{1}^{*} & 1 \\
\gamma_{2}^{*} & \rho_{2}^{*} & 1 \\
\vdots & \vdots & \vdots \\
\gamma_{n}^{*} & \rho_{n}^{*} & 1
\end{array}\right] \mathbf{F}\left[\begin{array}{cccc}
\gamma_{1} & \gamma_{2} & \cdots & \gamma_{n} \\
\rho_{1} & \rho_{2} & \cdots & \rho_{n} \\
1 & 1 & \cdots & 1
\end{array}\right]=\mathbf{0}
$$

rewritten the equation (2) we have

$$
\text { Af }=0
$$

where

$$
\mathbf{A}=\left[\begin{array}{ccccccccc}
\gamma_{1} \gamma_{1}^{*} & \gamma_{1} \rho_{1}^{*} & \gamma_{1} & \rho_{1} \gamma_{1}^{*} & \rho_{1} \rho_{1}^{*} & \rho_{1} & \gamma_{1}^{*} & \rho_{1}^{*} & 1 \\
\gamma_{2} \gamma_{2}^{*} & \gamma_{2} \rho_{2}^{*} & \gamma_{2} & \rho_{2} \gamma_{2}^{*} & \rho_{2} \rho_{2}^{*} & \rho_{2} & \gamma_{2}^{*} & \rho_{2}^{*} & 1 \\
& & & & \vdots & & & & \\
\gamma_{n} \gamma_{n}^{*} & \gamma_{n} \rho_{n}^{*} & \gamma_{n} & \rho_{n} \gamma_{n}^{*} & \rho_{n} \rho_{n}^{*} & \rho_{n} & \gamma_{n}^{*} & \rho_{n}^{*} & 1
\end{array}\right]
$$

and

$\mathbf{f}=\left[\begin{array}{lllllllll}F_{11} & F_{12} & F_{13} & F_{21} & F_{22} & F_{23} & F_{31} & F_{32} & F_{33}\end{array}\right]^{T}$

contains the coefficients of matrix $\mathbf{F}$.

There exist several methods to compute the fundamental matrix, which can be grouped into three categories: linear methods, iterative methods and robust methods [3]. In order to compute a robust fundamental matrix $\mathbf{F}$ we apply the RANdom SAmple Consensus (RANSAC) approach which uses the 7points algorithm to compute a initial fundamental matrix $\mathbf{F}$. This approach uses $\mathbb{Q}_{i}$ subsets $\left(i=1,2, \ldots, \mathbf{m}_{s}\right)$, which are composed by 7 pairs of correlated points $q_{i, j} \rightarrow q_{i, j}^{*}$ $(j=1,2, \ldots, 7)[5]$.

The 7-points algorithm approach starts with a singular value decomposition (SVD) of matrix $\mathbf{A}$ to solve the equation (3. As matrix $\mathbf{A}$ is formed only with 7 correlated points it has rank 7, then the right null space of matrix $\mathbf{A}$ is 2-dimensional. Consequently, we have two matrix $\mathbf{G}_{i, 1}$ and $\mathbf{G}_{i, 2}$. In order to computes the initial fundamental matrix $\mathbf{F}_{i}$ we set a linear convex combination as follows

$$
\mathbf{G}_{i}=\mu \mathbf{G}_{i, 1}+(1-\mu) \mathbf{G}_{i, 2}
$$

where $\mu \in[0,1]$. Considering the rank property of the fundamental matrix we have

$$
\operatorname{det}\left(\mu \mathbf{G}_{i, 1}+(1-\mu) \mathbf{G}_{i, 2}\right)=0
$$


from the equation (7) we obtain a polynomial $W(\mu)$ of degree 3 with roots $\left(\mu_{1}, \mu_{2}, \mu_{3}\right)$. Replacing the real part of $\mu_{1}, \mu_{2}$ and $\mu_{3}$ in (6) we have three candidates matrices, but we choice the candidate matrix with lower residual error as the initial fundamental matrix $\mathbf{F}_{i}$. Then all initial fundamental matrix are evaluated considering the criteria of Sampson distance and the initial fundamental matrix $\mathbf{F}_{i}$ with lower total error is retained as a fundamental matrix $\mathbf{F}_{r}$.

Using the estimate fundamental matrix $\mathbf{F}_{r}$ we remove all the spurious correlated points. Once removed all the spurious points, the robust fundamental matrix $\mathbf{F}$ is compute using the 8points method [16] which admits only one solution to equation (3). Robust fundamental matrix will be used later in the 3D reconstruction approach described later in III.

Another important matrix in the epipolar geometry theory is the essential matrix E. In fact this matrix is a generalization of fundamental matrix $\mathbf{F}$. The essential matrix is obtained as follows

$$
\mathbf{E}=\mathbf{K}^{* \mathbf{T}} \mathbf{F K}
$$

where $\mathbf{K}$ and $\mathbf{K}^{*}$ are the intrinsic parameter matrices of left and right camera respectively. Essential has two equal singular values and the third is zero. Essential matrix is a matrix with rank 2, Clearly, the essential matrix can be used only with a calibrated stereo ring. In order to compute the intrinsic parameters $\mathbf{K}$ and $\mathbf{K}^{*}$ from left and right cameras respectively and the extrinsic parameters $\tilde{\mathbf{R}}, \tilde{\mathbf{R}}^{*}, \mathbf{t}$ and $\mathbf{t}^{*}$ there exist several camera calibration methods as proposed in [5], [22] which is used in this case,employing the calibration information of each camara . Let $\tilde{\mathbf{R}}$ and $\tilde{\mathbf{R}}^{*}$ be the rotation matrices and $\mathbf{t}$ and $\mathbf{t}^{*}$ the translation vector for each camera.

\section{3D RECONSTRUCTION}

As we mentioned above, the $3 \mathrm{D}$ reconstruction is the second problem to be dealt in a the stereo vision system. In this subsection we present the euclidian 3D reconstruction approach to solve this problem [14]. Fundamental matrix $\mathbf{F}$ ha ve been computed using RANSAC approach and the set of correlated points are available (gravity center and vertices of a rectangle).

In order to avoid the problem of having no intersection of vectors $\overrightarrow{C q}$ and $\overrightarrow{C^{*} q^{*}}$ in the 3D space we apply a position correction method to correlated image points $q_{i} \rightarrow q_{i}^{*}$ [5] [19]. Where $q_{i}=(\gamma, \rho, 1)^{T}$ and $q_{i}^{*}=\left(\gamma^{*}, \rho^{*}, 1\right)^{T}$. Initially, the correction algorithm takes the correlated points to the origin of image plane, it means $\gamma=\rho=\gamma^{*}=\rho^{*}=0$. The matrices transformation to develop this translation are defined by

$$
\mathbf{T}_{a}=\left[\begin{array}{ccc}
1 & 0 & -\gamma \\
0 & 1 & -\rho \\
0 & 0 & 1
\end{array}\right] \quad \mathbf{T}_{a}^{*}=\left[\begin{array}{ccc}
1 & 0 & -\gamma^{*} \\
0 & 1 & -\rho^{*} \\
0 & 0 & 1
\end{array}\right]
$$

Using the matrice transformation $\mathbf{T}_{a}$ and $\mathbf{T}_{a}^{*}$ the initial fundamental matrix is replaced by

$$
\mathbf{F}_{n 1}=\mathbf{T}_{a}^{*-T} \mathbf{F} \mathbf{T}_{a}
$$

where $\mathbf{F}_{n 1}$ corresponds to fundamental matrix for the translated coordinates. Now, the left and right epipoles of $\mathbf{F}_{n 1}$ are defined by $\mathbf{F}_{n 1} e=0$ and $e^{* T} \mathbf{F}_{n 1}=0$ respectively, with $\mathbf{e}=$ $\left(e_{1}, e_{2}, e_{3}\right)$ and $\mathbf{e}^{*}=\left(e_{1}^{*}, e_{2}^{*}, e_{3}^{*}\right)$. Epipoles must be normalized, it means we have to multiply the epipole e by the scale factor $1 /\left(e_{1}^{2}+e_{2}^{2}\right)$ and do the same for $\mathbf{e}^{*}$ with the scale factor $1 /\left(e_{1}^{* 2}+e_{2}^{* 2}\right)$.

Then the fundamental matrix $\mathbf{F}_{n 1}$ once again is replaced by

$$
\mathbf{F}_{n 2}=\mathbf{R}_{a}^{*} \mathbf{F}_{n 1} \mathbf{R}_{a}^{T}=\left[\begin{array}{ccc}
\hat{f} \hat{f}^{*} d & -\hat{f}^{*} c & -\hat{f}^{*} d \\
\hat{f} b & a & b \\
-\hat{f} d & c & d
\end{array}\right]
$$

where $a, b, c, d, \hat{f}$ and $\hat{f}^{*}$ are a set of variables defining the relationship between the matrix entries. Furthermore

$$
\mathbf{R}_{a}=\left[\begin{array}{ccc}
e_{1} & e_{2} & 0 \\
-e_{2} & e_{1} & 0 \\
0 & 0 & 1
\end{array}\right] \quad \mathbf{R}_{a}^{*}=\left[\begin{array}{ccc}
e_{1}^{*} & e_{2}^{*} & 0 \\
-e_{2}^{*} & e_{1}^{*} & 0 \\
0 & 0 & 1
\end{array}\right]
$$

Consider an epipolar line $\mathbf{l}$ passing through $q=(0, t, 1)^{T}$ and the epipole $\mathbf{e}=(1,0, \hat{f})$. Then $\mathbf{l}=q \otimes \mathbf{e}$, so the squared distance from this line to the origin is

$$
d(q, \mathbf{l})^{2}=\frac{t^{2}}{1+(t \hat{f})^{2}}
$$

The corresponding epipolar line $\mathbf{l}^{*}$ is defined as

$$
\mathbf{l}^{*}=\mathbf{F}_{n 2} q=\left(-\hat{f}^{*}(c t+d), a t+b, c t+d\right)^{T}
$$

The square distance of the epipolar line (14) from the origin is given by

$$
d\left(q^{*}, \mathbf{l}^{*}\right)=\frac{(c t+d)^{2}}{(a t+b)^{2}+\hat{f}^{* 2}(c t+d)^{2}}
$$

therefore the total distance is

$$
s(t)=\frac{t^{2}}{1+(t \hat{f})^{2}}+\frac{(c t+d)^{2}}{(a t+b)^{2}+\hat{f}^{* 2}(c t+d)^{2}}
$$

the aim of this approach is to minimize the total distance, then we find the minimum of (16) using its derivative. The minimum occurs when $\frac{d s(t)}{d t}=0$, then

$$
\begin{aligned}
\epsilon(t)= & t\left((a t+b)^{2}+\hat{f}^{* 2}(c t+d)^{2}\right)^{2} \\
& -(a d-b c)\left(1+\hat{f}^{2} t^{2}\right)^{2}(a t+b)(c t+d)=0
\end{aligned}
$$

We assess the cost function (16) at real roots of $\epsilon(t)$ and we select the value $t_{\min }$ of $t$ that gives the smallest value of cost function. After that we evaluate the lines $\mathbf{l}=$ $(t \hat{f}, 1,-t)$ and $\mathbf{l}^{*}$ given in (14). We have to find the new pair of corresponding points $\hat{q} \rightarrow \hat{q}^{*}$ related to these lines. For a general line $\left(a_{l}, b_{l}, c_{l}\right)$ the closest point is given by $\left(-a_{l} c_{l},-b_{l} c_{l}, a_{l}^{2}+b_{l}^{2}\right)$. 
Once obtained this new set of corresponding points, they are transferred back to the original coordinates by replacing $\hat{q}$ by $\tilde{q}=\mathbf{T}_{a}^{-1} \mathbf{R}_{a}^{T} \hat{q}$ and $\hat{q}^{*}$ by $\tilde{q}^{*}=\mathbf{T}_{a}^{*-1} \mathbf{R}_{a}^{* T} \hat{q}^{*}$.

The $3 \mathrm{D}$ point $\tilde{p}=(\tilde{x}, \tilde{y}, \tilde{z}, s c)^{T}$ related with the corresponding image points $\tilde{q} \rightarrow \tilde{q}^{*}$ is computed using the SVD decomposition of following matrix equation

$$
\left[\begin{array}{c}
\gamma \mathbf{T}_{3}-\mathbf{T}_{1} \\
\rho \mathbf{T}_{3}-\mathbf{T}_{2} \\
\gamma^{*} \mathbf{T}_{3}^{*}-\mathbf{T}_{1}^{*} \\
\rho^{*} \mathbf{T}_{3}^{*}-\mathbf{T}_{2}^{*}
\end{array}\right]\left[\begin{array}{c}
x \\
y \\
z \\
s c
\end{array}\right]=\mathbf{0}
$$

this matrix equation comes from the fact that $q \otimes \mathbb{T} p=0$. Then the real point $p$ expressed in homogeneous coordinates is

$$
p=\frac{\tilde{p}}{s c}=\left[\frac{\tilde{x}}{s c}, \frac{\tilde{f}}{s c}, \frac{\tilde{z}}{s c}, 1\right]^{T}=[x, y, z, 1]^{T}
$$

\section{SySTEM CONCEPT}

The platform is composed of an eight-rotor aircraft which has 2 RABBIT microprocessor RCM3400 on board. This microprocessor has the following main features: module running at $29.4 \mathrm{MHz}, 512 \mathrm{~K}$ flash memory, $4 \mathrm{PWM}$ outputs, 6 serial ports, 2 input capture channels. The microprocessor 1 runs the control algorithm in real-time to control the attitude and the altitud of the eight-rotor rotorcraft, therefore it reads the information provided by the IMU sensor. The second one microprocessor is used to compute the PWM level output to control the lateral rotors, using the information provided by the stereo vision system ( $x-y$ position). This information arrives to this microprocessor by modem.

The stereo vision system is composed by two cameras Logitech Pro5000 (webcam), this cameras are configured to optimize light variations and color balancing, with an image resolution of $320 \times 240$ pixels. The images captured by the on-board cameras is sent to a PC on the ground through a USB connection. The frames of the images are treated in the computer devoted to vision. This computer obtains the 3D information which is sent to microprocessor 2 by modem. The vision algorithms are developed in $\mathrm{C}++$ using OpenCV library, 18 frames per seconds in each camera are necessary to obtain the result to calculate the $3 \mathrm{D}$ pose.

A Microbotics IMU is used to measure the angular rate, the acceleration, and the direction of the earth magnetic field with a sample rate of up to $50 \mathrm{~Hz}$. It is composed by 3 -axis gyro with range of $\pm 300^{\circ} / \mathrm{sec}, 3$-axis accelerometer with range of $\pm 6 g$ and 3 -axis magnetometer.

\section{Dynamical Model of UAV}

The aerial robot under consideration consists of a rigid cross frame equipped with eight rotors as shown in Figure 4. $\psi$ is the yaw angle around the $z$ axis, $\theta$ is the pitch angle around the $y$ axis, and $\phi$ is the roll angle around the $x$ axis. In order to avoid the yaw drift due to the reactive torques, the main rotors are such that the right and left rotors rotate clockwise while the front and rear rotors rotate counterclockwise. Similarly the external lateral motors located on the same axis rotate in

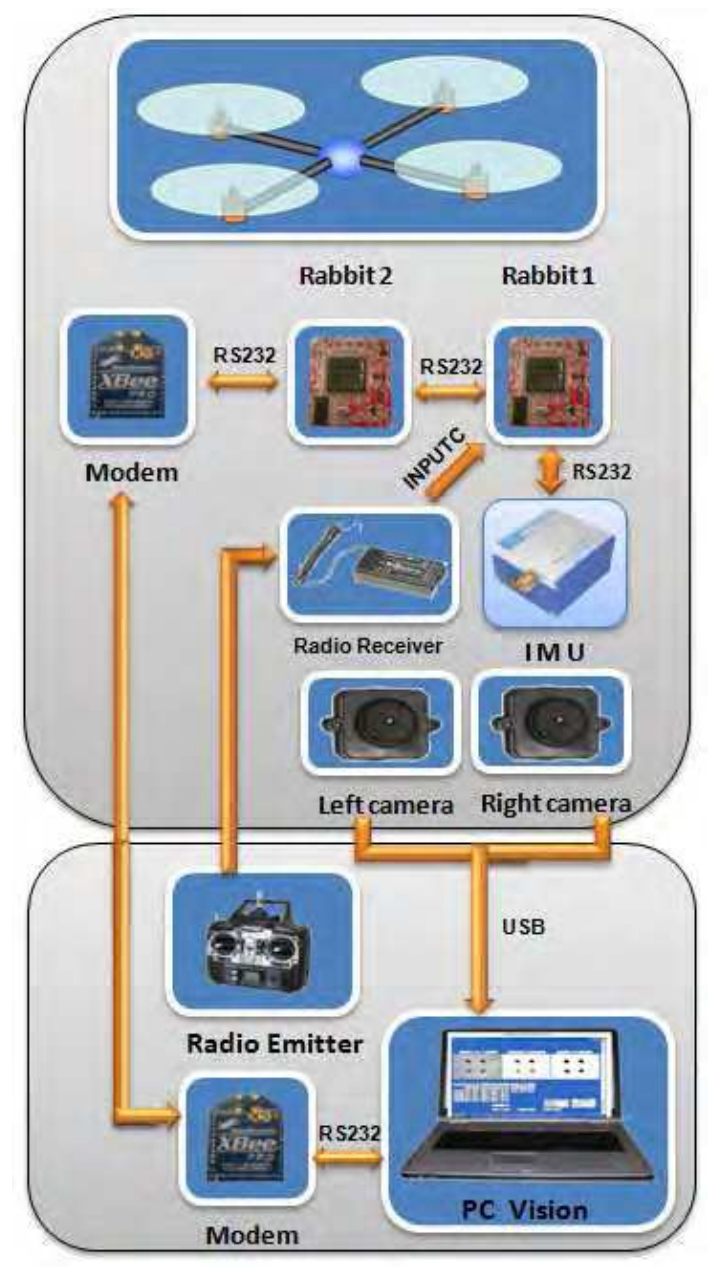

Fig. 3. Architecture scheme use in the Eight-rotor rotorcraft.

opposite directions to avoid interacting with the roll and pitch displacements.

M1 through M4 in Figure 4 are the front, right, rear and left main motors respectively and they are devoted to stabilize the UAV. While the lateral motors motors M5 through M8 follow the same order of location and they perform the lateral displacements. The speed relationships between speed of motors (main and lateral) to perform angular and translational displacements in 3D space are given in [18].

In order to model the dynamic system, we define a couple of coordinate frames. Let $\mathcal{I}=\{\vec{i}, \vec{j}, \vec{k}\}$ be an external reference set of axis, and let $\mathcal{B}=\{\vec{\imath}, \vec{j}, \overrightarrow{\vec{k}}\}$ denote a set of coordinates fixed to the rigid aircraft as is shown in Figure 4.

The dynamical model of the aircraft will be obtained using the Newton-Euler approach [4]. The dynamical model can be expressed by the following equations

$$
\begin{aligned}
\dot{\xi} & =v \\
m \dot{v} & =\bar{f} \\
\dot{\mathbf{R}} & =\mathbf{R} \hat{\Omega} \\
\mathbb{J} \dot{\Omega} & =-\Omega \times \mathbb{J} \Omega+\tau
\end{aligned}
$$

where $v=\dot{\xi} \in \mathbb{R}^{3}$ is the body's velocity in the frame $\mathcal{I}, \mathbf{R}$ 


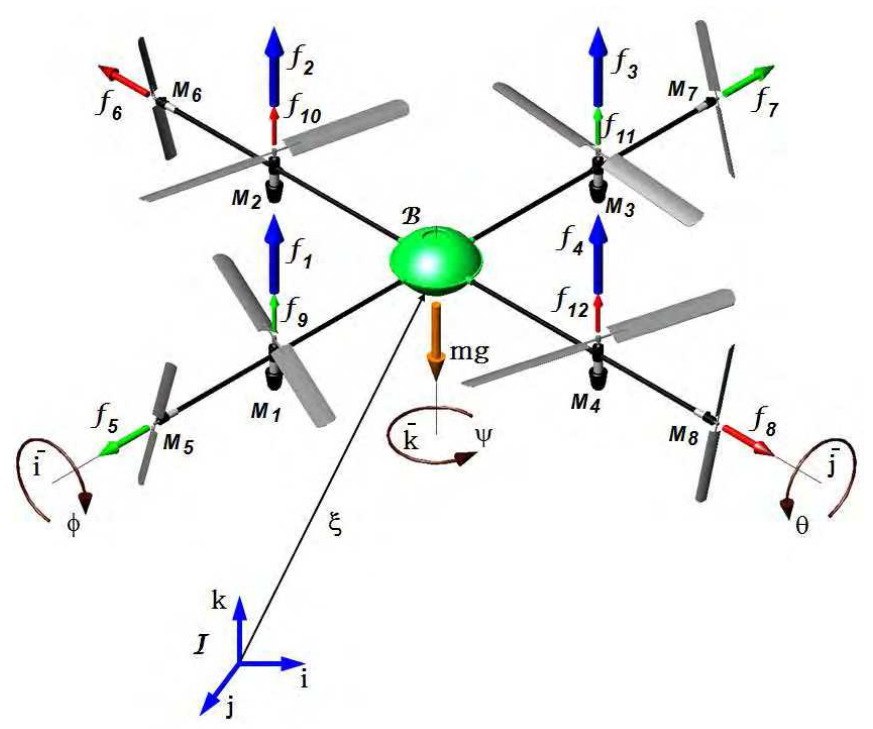

Fig. 4. Eight-rotor rotorcraft scheme. Where $f_{i}$ is the force produced by the motor $M_{i}$ with $i=1,2, \ldots, 8$ and $f_{j}$ with $j=9,10,11,12$ are the additional forces produced by the lateral motors.

represents the rotation matrix. This rotation matrix represents the orientation of body frame $\mathcal{B}$ with respect to the inertial frame $\mathcal{I} . \bar{f}$ denotes the external force applied to center mass $m_{c}$ with respect to inertial frame $\mathcal{I}$. This vector includes the gravitational force, the control inputs related with the translational displacements and the drag forces [2][1]. $\tau$ denotes the vector composed by the external torques applied to $m_{c}$, it is referred to frame $\mathcal{B}$. The vector $\Omega \in \mathbb{R}^{3}$ describes the angular velocity and the matrix $\mathbb{J} \in \mathbb{R}^{3 \times 3}$ acts as the inertia matrix. Let $\hat{\Omega}$ be the skew-symmetrical matrix of vector $\Omega$, which is defined as

$$
\hat{\Omega}=\left(\begin{array}{ccc}
0 & -\Omega^{3} & \Omega^{2} \\
\Omega^{3} & 0 & -\Omega^{1} \\
-\Omega^{2} & \Omega^{1} & 0
\end{array}\right)
$$

Rotational dynamics must be expressed in the body frame $\mathcal{B}$. Defining the attitude vector $\eta=\left[\begin{array}{lll}\psi & \theta & \psi\end{array}\right]^{T}$ as

$$
\dot{\eta}=W_{\eta}^{-1} \Omega
$$

where $W_{\eta}$ represents the Jacobian matrix defined in [4]. Then

$$
\Omega=\left[\begin{array}{c}
\dot{\phi}-\dot{\psi} s_{\theta} \\
\dot{\theta} c_{\phi}+\dot{\psi} c_{\theta} s_{\phi} \\
\dot{\psi} c_{\theta} c_{\phi}-\dot{\theta} s_{\phi}
\end{array}\right] .
$$

In this model, dynamical equation could be naturally separated into translational and rotational subsystems. That couple of subsystems will be present in next subsections.

\section{A. Translational subsystem model}

To develop the dynamical model of the flying machine we consider the forces that come from three different sources: inertia, air drag and gravity [2][4]. They are opposed to translational and rotational motions.
Using the Newton-Euler approach the translational subsystem can be expressed as

$$
\begin{aligned}
m \dot{v} & =\bar{f} \\
m \ddot{\xi} & =F_{p}+F_{d}+F_{g}
\end{aligned}
$$

where $F_{p}$ defines the force produced by the propeller system, $F_{d}$ is the vector of forces due to drag, $F_{g}$ represents the gravity force, $m$ is the mass of the vehicle and $\xi=[x, y, z]^{T}$ is the position with respect to $\mathcal{I}$. The force $F_{p}=\left[f_{x}, f_{y}, f_{z}\right]^{T}$ expressed in the frame $\mathcal{B}$ is given by

$$
F_{p}^{\mathcal{B}}=\left[\begin{array}{l}
u_{x} \\
u_{y} \\
u_{z}
\end{array}\right]=\left[\begin{array}{c}
f_{5}-f_{7} \\
f_{6}-f_{8} \\
\sum_{i=1}^{4} f_{i}+\sum_{j=9}^{12} f_{j}
\end{array}\right]
$$

where the forces $f_{i}(i=1, \ldots, 8)$ are the forces generate for the propeller $M_{i}$. While the forces $f_{j}(j=9, \ldots, 12)$ are the additional lift forces acting over each main rotor [18]. It means that the magnitude of those additional vectors is a function of the incoming lateral air flow. Then the vector $F_{p}$ with respect to inertial frame is obtained by

$$
F_{p}=\mathbf{R} F_{p}^{\mathcal{B}}
$$

where $\mathbf{R}$ is the rotation matrix representing the orientation of the rotorcraft from $\mathcal{B}$ to $\mathcal{I}$. We use $c_{\theta}$ to denote $\cos \theta$ and $s_{\theta}$ for $\sin \theta$

$$
\mathbf{R}=\left[\begin{array}{ccc}
c_{\psi} c_{\theta} & c_{\psi} s_{\theta} s_{\phi}-s_{\psi} c_{\phi} & c_{\psi} s_{\theta} c_{\phi}+s_{\psi} s_{\phi} \\
s_{\psi} c_{\theta} & s_{\psi} s_{\theta} s_{\phi}+c_{\psi} c_{\phi} & s_{\psi} s_{\theta} c_{\phi}-c_{\psi} s_{\phi} \\
-s_{\theta} & c_{\theta} s_{\phi} & c_{\theta} c_{\phi}
\end{array}\right]
$$

Let $F_{d}$ be the drag vector, as well is known the drag force experienced by the UAV is related with the translational speed. Then the drag vector is defined as

$$
F_{d}=K_{d} \dot{\eta}
$$

where $K_{d}=\operatorname{diag}\left[k_{d x}, k_{d y}, k_{d z}\right]$ is the matrix which contains the translational drag coefficients [2]. Finally the gravity force $F_{g}$ acts only on the $z$-axis, then this force is represented by

$$
F_{g}=m\left[\begin{array}{lll}
0 & 0 & g
\end{array}\right]^{T}=m \mathbf{g}
$$

In order to model the additional lift forces $f_{j}(j=$ $9,10,11,12)$ acting on the rotorcraft, we consider the control input $u, u_{x}, u_{y}$. According to equation (22) we have

$$
\begin{aligned}
& u_{x}=f_{5}-f_{7}=u_{x_{1}}-u_{x_{2}} \\
& u_{y}=f_{8}-f_{6}=u_{y_{1}}-u_{y_{2}} \\
& u_{z}=u+f_{9}+f_{10}+f_{11}+f_{12}
\end{aligned}
$$

where $u_{x_{1}}$ and $u_{x_{2}}$ are the control inputs for the front motor and the rear motor in $x$-axis respectively. $u_{y_{1}}$ and $u_{y_{2}}$ are defined similarly for the left motor and right motor in $y$-axis. While $u$ is defined as follows

$$
u=f_{1}+f_{2}+f_{3}+f_{4}
$$




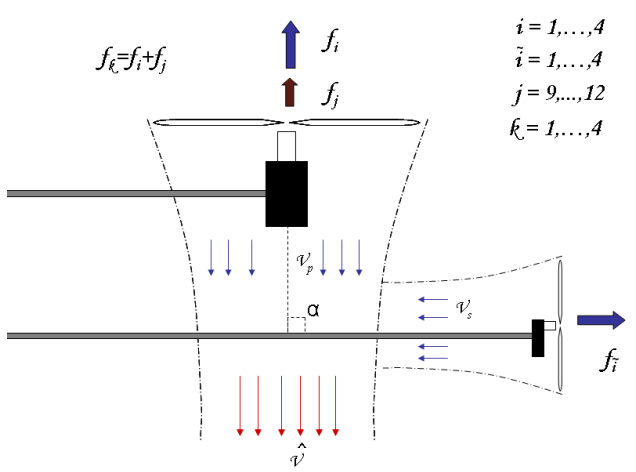

Fig. 5. Analysis of the main and lateral thrusts.

with

$$
f_{i}=k_{i} \omega_{i}^{2}, \quad i=1, \ldots, 8
$$

where $k_{i}>0$ is a parameter depending on the density of air, the radius, the shape, the pitch angle of the blade and $\omega_{i}$ is the angular speed of the each motor " $i$ " $\left(M_{i}, i=1, \ldots, 8\right)$. There exist additional forces $f_{9}$ to $f_{12}$ acting on each one of the four main rotors, see Figure 5. These forces are due to the airflow generated by the lateral rotors. It means that the magnitude of vectors $f_{9}$ to $f_{12}$ are functions of the lateral air flow produced by the corresponding lateral rotor. The induced wind speed in a propeller is defined as follows

$$
V=\left(\frac{f}{2 \rho A}\right)^{\frac{1}{2}}
$$

where $f$ is the thrust generated by the propeller, $\rho$ is the air density and $A$ is the propeller area [12]. In order to clarify the notation we will use the subscripts $p$ for the main rotor and subscript $s$ for the lateral rotor. The thrust $f$ provided the main rotor in combination with a lateral rotor can be expressed as

$$
f_{p}=2 \rho A_{p} \hat{V} V_{p}
$$

where $V_{p}$ is the induced wind speed in the main propeller and $\hat{V}$ is the total induced wind speed by the set of rotors, this is given by

$$
\hat{V}=\left[\left(V_{s} \cos \alpha+V_{p}\right)^{2}+\left(V_{s} \sin \alpha\right)^{2}\right]^{\frac{1}{2}}
$$

where $\alpha$ is the angle between the main rotor axis and the lateral rotor axis. It is important to notice that without extra lateral rotor $V_{s}=0$, this implies that $\hat{V}=V_{p}$, and (31) becomes

$$
f_{p}=2 \rho A_{p} V_{p}^{2}
$$

Introducing (32) into (31) with $\alpha=90^{\circ}$ we obtain

$$
f_{p}=2 \rho A_{p} V_{p}^{2}\left(1+\frac{V_{s}^{2}}{V_{p}^{2}}\right)^{\frac{1}{2}}
$$

The additional nonlinear term $\left(V_{s}^{2} / V_{p}^{2}\right)$ appearing in this equation is related to the airflow produced by the corresponding lateral rotor, which does not appear in (33). Nevertheless, this extra term has an almost linear behavior mainly for large values of $V_{s}$. In practice, we consider the parameter $V_{p}$ as constant at hover, since this parameter depends on the thrust generated by the main rotor to stabilize the mini-helicopter. In our experiment, we work in a region where the relationship $\left(\frac{V_{s}}{V_{p}}\right) \leq 1$ holds. It then follows

$$
\left(1+\frac{V_{s}^{2}}{V_{p}^{2}}\right)^{\frac{1}{2}} \leq \sqrt{2}
$$
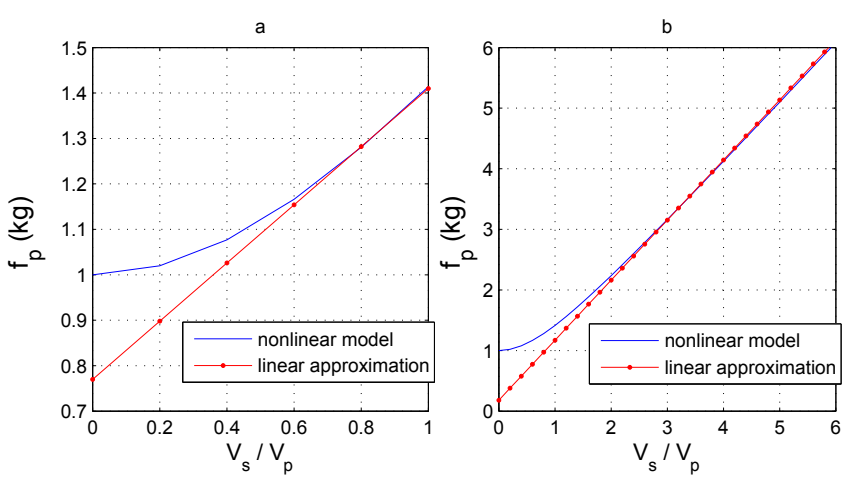

Fig. 6. Main thrust behavior with incoming lateral wind due to lateral propeller

The thrust behavior with respect to $\left(V_{s} / V_{p}\right)$ is shown in Figure 6. Figure 6a shows the region defined by $V_{s} \leq V_{p}$ where the nonlinear model is approximated by a straight line with slope $\bar{m}=0.64$ which intercepts the vertical axis at $y_{b}=0.77$. Figure $6 \mathrm{~b}$ shows the nonlinear behavior of (35) when $V_{s}>V_{p}$ which is approximately a straight line with slope $\bar{m}=1$ without drift in the vertical axis.

The additional term in (34) will be compensated to effectively decouple the translational and rotational displacements.

The force in each one of the four main rotors is affected by the lateral thrust $f_{s}$ of the corresponding lateral rotor. The lateral thrust in turn depends on the control actions $u_{x}$ and $u_{y}$ applied to lateral motors. Considering identical lateral motors, forces $f_{9}$ to $f_{12}$ can be expressed as follows

$$
f_{9}=b u_{x_{1}}, \quad f_{10}=b u_{y_{2}}, \quad f_{11}=b u_{x_{2}}, \quad \text { and } \quad f_{12}=b u_{y_{1}}
$$

where $b \geq 0$ is the approximate slope in Figure 6a. Then, the force vector $F_{b}^{\mathcal{B}}$ can be rewritten as follows

$$
F_{b}^{\mathcal{B}}=\left[\begin{array}{c}
u_{x} \\
u_{y} \\
u_{z}
\end{array}\right]=\left[\begin{array}{c}
u_{x} \\
u_{y} \\
u+b \bar{u}
\end{array}\right]
$$

where $\bar{u}=u_{x_{1}}+u_{y_{2}}+u_{x_{2}}+u_{y_{1}}$.

Introducing (23), (25) and (26) in (21), we obtain 


$$
\begin{aligned}
m \ddot{x}= & u_{x} c_{\theta} c_{\psi}-u_{y}\left(c_{\phi} s_{\psi}-c_{\psi} s_{\theta} s_{\phi}\right) \\
& +u_{z}\left(s_{\phi} s_{\psi}+c_{\phi} c_{\psi} s_{\theta}\right)+k_{d x} \dot{x} \\
m \ddot{y}= & u_{x} c_{\theta} s_{\psi}+u_{y}\left(c_{\phi} c_{\psi}+s_{\theta} s_{\phi} s_{\psi}\right) \\
& -u_{z}\left(c_{\psi} s_{\phi}-c_{\phi} s_{\theta} s_{\psi}\right)+k_{d y} \dot{y} \\
m \ddot{z}= & -u_{x} s_{\theta}+u_{y} c_{\theta} s_{\phi}-m g \\
& +u_{z} c_{\theta} c_{\phi}+k_{d z} \dot{z}
\end{aligned}
$$
UAV.

This equation represents the translational dynamic of the

\section{B. Rotational subsystem model}

In order to obtain a model with respect to rotational dynamic of the rotorcraft, the Newton's law about the rotation motion is used. The sum of moments is given by

$$
\tau_{p}-\tau_{a}-\tau_{g}=\mathbb{J} \dot{\Omega}+\Omega \times \mathbb{J} \Omega
$$

where $\tau_{p}$ is the rotational moment produced by the main propellers with respect to body fixed frame, $\tau_{a}$ is the aerodynamic friction torque and $\tau_{g}$ represents the gyroscopic torque. The inertia matrix is represented by $\mathbb{J}$ and $\Omega$ is the angular speed vector. Considering that the flying machine is symmetrical and assuming that the coupling inertia is zero, the inertia matrix is a diagonal matrix defined as follows

$$
\mathbb{J}=\operatorname{diag}\left[\begin{array}{lll}
\mathbf{I}_{x}, & \mathbf{I}_{y}, & \mathbf{I}_{z}
\end{array}\right]
$$

where each term of this matrix is given in a general form by $\mathbf{I}_{()}=\mathbf{I}_{c()}+\mathbf{I}_{M()}+\mathbf{I}_{m()}+\mathbf{I}_{b()}$. Right hand terms represent the inertia due to carbon fiber cross, main motors, lateral motors and to the battery. First, we will model the cross with tow slender rods that intersect, the body frame axes are coincident with the arms of the cross. $x$ - axis points on the forward arm and $y-$ axis points on left arm. The inertia of the cross for rotation with respect to intersection is

$$
\begin{aligned}
\mathbf{I}_{c x} & =\mathbf{I}_{c y}=\frac{3}{4} m_{c} r_{c}^{2}+\frac{1}{12} m_{c} l^{2} \\
\mathbf{I}_{c z} & =\frac{1}{2} m_{c} r_{c}^{2}+\frac{1}{6} m_{c} l^{2}
\end{aligned}
$$

where $m_{c}$ is the mass of the rods, $l=2 l_{c}$ and $r_{c}$ is radius of the cross pieces. In order to obtain the inertia coefficient of the main motors, we will consider them as a cylinders of radius $r_{m}$, length $h_{m}$ and mass $m_{m}$. They are located with a distance $l_{c}$ from the interception point in the middle of each arm of the cross. The inertia for the motors $M_{1}$ and $M 3$ located on the $x$-axis is given by

$$
\begin{aligned}
& \mathbf{I}_{M_{1} x}=\mathbf{I}_{M_{3} x}=\frac{1}{4} m_{m} r_{m}^{2}+\frac{1}{3} m_{m} h_{m}^{2} \\
& \mathbf{I}_{M_{1} y}=\mathbf{I}_{M_{3} y}=\frac{1}{4} m_{m} r_{m}^{2}+\frac{1}{3} m_{m} h_{m}^{2}+m_{m} l_{c}^{2} \\
& \mathbf{I}_{M_{1} z}=\mathbf{I}_{M_{3} z}=\frac{1}{2} m_{m} r_{m}^{2}+m_{m} l_{c}^{2}
\end{aligned}
$$

Now, the inertia coefficients for the motors $M_{2}$ and $M_{4}$ located on $y$-axis are given by

$$
\begin{aligned}
\mathbf{I}_{M_{2} x} & =\mathbf{I}_{M_{4} x}=\frac{1}{4} m_{m} r_{m}^{2}+\frac{1}{3} m_{m} h_{m}^{2}+m_{m} l_{c}^{2} \\
\mathbf{I}_{M_{2} y} & =\mathbf{I}_{M_{4} y}=\frac{1}{4} m_{m} r_{m}^{2}+\frac{1}{3} m_{m} h_{m}^{2} \\
\mathbf{I}_{M_{2} z} & =\mathbf{I}_{M_{4} z}=\frac{1}{2} m_{m} r_{m}^{2}+m_{m} l_{c}^{2}
\end{aligned}
$$

Considering that the pair of lateral motors $M_{5}-M_{7}$ is located on the $x$-axis and the pair $M_{6}-M_{8}$ on the $y$-axis. Then the inertia coefficients for these motor are given by

$$
\begin{aligned}
& \mathbf{I}_{\mathbf{m}_{5} x}=\mathbf{I}_{\mathbf{m}_{6} y}=\mathbf{I}_{\mathbf{m}_{7} x}=\mathbf{I}_{\mathbf{m}_{8} y}=\frac{1}{2} m_{a} r_{a}^{2} \\
& \mathbf{I}_{\mathbf{m}_{5} y}=\mathbf{I}_{\mathbf{m}_{6} x}=\mathbf{I}_{\mathbf{m}_{7} y}=\mathbf{I}_{\mathbf{m}_{8} x}=\delta \\
& \mathbf{I}_{\mathbf{m}_{5} z}=\mathbf{I}_{\mathbf{m}_{6} z}=\mathbf{I}_{\mathbf{m}_{7} z}=\mathbf{I}_{\mathbf{m}_{8} z}=\delta
\end{aligned}
$$

where $\delta=\frac{1}{4} m_{a} r_{a}^{2}+\frac{1}{3} m_{a} h_{a}^{2}+m_{a} l_{a}^{2}$. Furthermore, the parameters of the auxiliar motor are: radius $r_{a}$, length $h_{a}$, mass $m_{a}$ and distance from the intersection point in the middle of each arm of the cross to the center of mass of the auxiliar motors.

The inertia due to the battery is described by

$$
\begin{aligned}
& \mathbf{I}_{b x}=m_{b}\left(\frac{b_{w}^{2}+b_{h}^{2}}{12}\right)+m_{b} l_{0}^{2} \\
& \mathbf{I}_{b y}=m_{b}\left(\frac{b_{a}^{2}+b_{h}^{2}}{12}\right)+m_{b} l_{0}^{2} \\
& \mathbf{I}_{b z}=m_{b}\left(\frac{b_{w}^{2}+b_{a}^{2}}{12}\right)
\end{aligned}
$$

where $b_{a}, b_{w}, b_{h}$ are the battery dimensions, $m_{b}$ is its mass and $l_{0}$ represents the distance between the gravity center of the battery and the origin of body frame $\mathcal{B}$.

From the equations (39)-(43), the inertia about each axis is computed by summation. It means

$$
\begin{aligned}
& \mathbf{I}_{x}=\mathbf{I}_{c x}+\sum_{i=1}^{4} \mathbf{I}_{M_{i} x}+\sum_{i=5}^{8} \mathbf{I}_{\mathbf{m}_{i} x}+\mathbf{I}_{b x} \\
& \mathbf{I}_{y}=\mathbf{I}_{c y}+\sum_{i=1}^{4} \mathbf{I}_{M_{i} y}+\sum_{i=5}^{8} \mathbf{I}_{\mathbf{m}_{i} y}+\mathbf{I}_{b y} \\
& \mathbf{I}_{z}=\mathbf{I}_{c z}+\sum_{i=1}^{4} \mathbf{I}_{M_{i} z}+\sum_{i=5}^{8} \mathbf{I}_{\mathbf{m}_{i} z}+\mathbf{I}_{b z}
\end{aligned}
$$

$\tau_{p}$ is given by

$$
\tau_{p}=\left[\begin{array}{c}
\tau_{\psi} \\
\tau_{\theta} \\
\tau_{\phi}
\end{array}\right]+\left[\begin{array}{c}
0 \\
\tau_{x} \\
\tau_{y}
\end{array}\right]=\tau+\Delta \tau_{x, y}
$$

where the terms on right hand side are

$$
\begin{gathered}
\tau=\left[\begin{array}{c}
\tau_{M_{1}}-\tau_{M_{2}}+\tau_{M_{3}}-\tau_{M_{4}} \\
l_{c}\left(f_{2}-f_{4}\right) \\
l_{c}\left(f_{1}-f_{3}\right)
\end{array}\right] \\
\Delta \tau_{x, y}=\left[\begin{array}{c}
0 \\
\tau_{\mathbf{m}_{5}}-\tau_{\mathbf{m}_{7}} \\
\tau_{\mathbf{m}_{6}}-\tau_{\mathbf{m}_{8}}
\end{array}\right]=\left[\begin{array}{c}
0 \\
k_{a} l_{c}\left(f_{5}-f_{7}\right) \\
k_{a} l_{c}\left(f_{8}-f_{6}\right)
\end{array}\right]=\left[\begin{array}{c}
0 \\
b u_{x} \\
b u_{y}
\end{array}\right]
\end{gathered}
$$


where $\tau$ is the generalized torque vector (yaw, roll and pitch moments), $\Delta \tau_{x, y}$ is the generalized torque vector produced by lateral rotors $\mathbf{m}_{i}$ in the axis $x$ and $y$ and $k_{a}$ is a relational constant between the force and the torque in each rotor. $\tau_{(\cdot)_{i}} \forall i=1, \ldots 8$ is the torque resultant by the rotor $M_{i}\left(\mathbf{m}_{i}\right)$. Using the second Newton's law and neglecting the air friction the following equation is obtained

$$
I_{M i} \dot{\omega}_{i}=-k_{r} \omega_{i}^{2}+\tau_{M_{i}}
$$

where $I_{M i}$ is the angular moment of $i$ rotor and $k_{r}$ is the rotational coefficient. In hover mode this equation gives

$$
\tau_{M_{i}}=k_{r} \omega_{i}^{2} \quad i=1, \ldots, 8 .
$$

The torque due the aerodynamical friction $\tau_{f}$ could be expressed

$$
\tau_{f}=k_{f} \Omega
$$

where $k_{f}=\operatorname{diag}\left[k_{f x}, k_{f y}, k_{f z}\right]$ are the aerodynamical friction coefficients. Finally the rotational dynamics is given as follows

$$
\begin{aligned}
\tau_{p}-\tau_{f}-\tau_{g} & =\mathbb{J} W_{\eta} \ddot{\eta}+\mathbb{J} \dot{W}_{\eta} \dot{\eta}+W_{\eta} \dot{\eta} \times \mathbb{J} W_{\eta} \dot{\eta} \\
\tau+\Delta \tau_{x, y}-\tau_{f}-\tau_{g} & =\mathbb{J} W_{\eta} \ddot{\eta}+\mathbb{C}(\eta, \dot{\eta}) \dot{\eta} \\
\mathbb{J} W_{\eta} \ddot{\eta} & =\tau+\Delta \tau_{x, y}-\tau_{f}-\tau_{g}-\mathbb{C}(\eta, \dot{\eta}) \dot{\eta}
\end{aligned}
$$

where $\mathbb{C}(\eta, \dot{\eta})$ is the Coriolis matrix.

Therefore the complete dynamical model representing the eight-rotors rotorcraft is

$$
\begin{aligned}
m \ddot{x}= & u_{x} c_{\theta} c_{\psi}-u_{y}\left(c_{\phi} s_{\psi}-c_{\psi} s_{\theta} s_{\phi}\right) \\
& +u_{z}\left(s_{\phi} s_{\psi}+c_{\phi} c_{\psi} s_{\theta}\right)+k_{d x} \dot{x} \\
m \ddot{y}= & u_{x} c_{\theta} s_{\psi}+u_{y}\left(c_{\phi} c_{\psi}+s_{\theta} s_{\phi} s_{\psi}\right) \\
& -u_{z}\left(c_{\psi} s_{\phi}-c_{\phi} s_{\theta} s_{\psi}\right)+k_{d y} \dot{y} \\
m \ddot{z}= & -u_{x} s_{\theta}+u_{y} c_{\theta} s_{\phi}-m g \\
& +u_{z} c_{\theta} c_{\phi}+k_{d z} \dot{z} \\
\mathbb{J} W_{\eta} \ddot{\eta}= & \tau+\Delta \tau_{x, y}-\tau_{f}-\tau_{g}-\mathbb{C}(\eta, \dot{\eta}) \dot{\eta}
\end{aligned}
$$

\section{Control Strategy}

In this section we present a simple linear control law for the attitude stabilization and position of the eight-rotor aircraft. We are able to apply this kind of linear control strategy due to the decoupling between the rotational dynamics and translational dynamics of this UAV configuration. In order to further simplify the analysis and since $\mathbb{J}$ is nonsingular, let us consider the following linearizing control law

$$
\tau=\tau_{f}+\tau_{g}+\mathbb{C}(\eta, \dot{\eta}) \dot{\eta}+\mathbb{J} W_{\eta}\left(\tilde{\tau}+\Delta \tau_{x, y}\right)-\Delta \tau_{x, y}
$$

where

$$
\tilde{\tau}=\left[\begin{array}{lll}
\tilde{\tau}_{\psi} & \tilde{\tau}_{\theta} & \tilde{\tau}_{\phi}
\end{array}\right]^{T}
$$

are the new inputs. Introducing (48) in (47) we have

$$
\ddot{\eta}=\tilde{\tau}+\Delta \tau_{x, y}
$$

Rewriting (44)-(47) gives

$$
\begin{aligned}
m \ddot{x}= & u_{x} c_{\theta} c_{\psi}-u_{y}\left(c_{\phi} s_{\psi}-c_{\psi} s_{\theta} s_{\phi}\right) \\
& +(u+b \bar{u})\left(s_{\phi} s_{\psi}+c_{\phi} c_{\psi} s_{\theta}\right)+k_{d x} \dot{x} \\
m \ddot{y}= & u_{x} c_{\theta} s_{\psi}+u_{y}\left(c_{\phi} c_{\psi}+s_{\theta} s_{\phi} s_{\psi}\right) \\
& -(u+b \bar{u})\left(c_{\psi} s_{\phi}-c_{\phi} s_{\theta} s_{\psi}\right)+k_{d y} \dot{y} \\
m \ddot{z}= & -u_{x} s_{\theta}+u_{y} c_{\theta} s_{\phi}-m g \\
& +(u+b \bar{u}) c_{\theta} c_{\phi}+k_{d z} \dot{z} \\
\ddot{\psi}= & \tilde{\tau}_{\psi} \\
\ddot{\theta}= & \tilde{\tau}_{\theta}+b u_{x} \\
\ddot{\phi}= & \tilde{\tau}_{\phi}+b u_{y}
\end{aligned}
$$

where $u_{x}$ and $u_{y}$ are the control inputs devoted to develop lateral displacements, $u$ is the main thrust acting on $z$-axis of $\mathcal{B}$, and $\tilde{\tau}_{\psi}, \tilde{\tau}_{\theta}$ and $\tilde{\tau}_{\phi}$ are the new angular moments (yawing moment, pitching moment and rolling moment).

\section{A. Attitude control}

The control of the attitude can be obtained by using the following PD controllers:

$$
\begin{aligned}
\tilde{\tau}_{\psi} & =\sigma_{a}\left(-a_{1} \dot{\psi}-a_{2}\left(\psi-\psi_{d}\right)\right) \\
\tilde{\tau}_{\theta} & =\sigma_{a}\left(-a_{3} \dot{\theta}-a_{4} \theta\right)-b u_{x} \\
\tilde{\tau}_{\phi} & =\sigma_{a}\left(-a_{5} \dot{\phi}-a_{6} \phi\right)-b u_{y}
\end{aligned}
$$

where $\sigma_{p}$ is a saturation defined as

$$
\sigma_{p}(s)=\left\{\begin{array}{rll}
p & \text { if } & s>p \\
s & \text { if } & -p \leq s \leq p \\
-p & \text { if } & s<-p
\end{array}\right.
$$

Introducing $(57)-(59)$ into $(54)-(56)$, we obtain

$$
\begin{aligned}
\ddot{\psi} & =\sigma_{a}\left(-a_{1} \dot{\psi}-a_{2}\left(\psi-\psi_{d}\right)\right) \\
\ddot{\theta} & =\sigma_{a}\left(-a_{3} \dot{\theta}-a_{4} \theta\right) \\
\ddot{\phi} & =\sigma_{a}\left(-a_{5} \dot{\phi}-a_{6} \phi\right)
\end{aligned}
$$

where $a_{i}$ are positive constants such that the polynomials $s^{2}+$ $a_{i} s+a_{i+1}$ are stable (for $i=1, \ldots, 6$ ). In practice, the control parameters $a_{i}$ for $i=1, \ldots, 6$, are chosen to obtain a critically damped controller (See proof below).

\section{B. Horizontal displacements and altitude control}

Note that from $(61)-(63) \psi, \theta, \phi \rightarrow 0$. For a time $T$ large enough $\psi, \theta$ and $\phi$ are arbitrarily small, therefore, (51), (52) and $(53)$ reduce to

$$
\begin{aligned}
& m \ddot{x}=u_{x}+k_{d x} \dot{x} \\
& m \ddot{y}=u_{y}+k_{d y} \dot{y} \\
& m \ddot{z}=u-m g+b \bar{u}+k_{d z} \dot{z}
\end{aligned}
$$

Let us propose the following control inputs 


$$
\begin{aligned}
u_{x} & =-m \sigma_{b}\left(b_{1} \dot{x}+b_{2}\left(x-x_{d}\right)\right)-k_{d x} \dot{x} \\
u_{y} & =-m \sigma_{b}\left(b_{3} \dot{y}+b_{4}\left(y-y_{d}\right)\right)-k_{d y} \dot{y} \\
u & =-m \sigma_{b}\left(b_{5} \dot{z}+b_{6}\left(z-z_{d}\right)\right)+m g-b \bar{u}-k_{d z} \dot{z}
\end{aligned}
$$

then the translational dynamics (64)-(66) becomes

$$
\begin{aligned}
& \ddot{x}=-\sigma_{b}\left(b_{1} \dot{x}+b_{2}\left(x-x_{d}\right)\right) \\
& \ddot{y}=-\sigma_{b}\left(b_{3} \dot{y}+b_{4}\left(y-y_{d}\right)\right) \\
& \ddot{z}=-\sigma_{b}\left(b_{5} \dot{z}+b_{6}\left(z-z_{d}\right)\right)
\end{aligned}
$$

where $x_{d}, y_{d}$ and $z_{d}$ are the coordinates of the desired position of the multi-rotor aircraft. As in (61)-(63), the control parameters $b_{i}$ for $i=1, \ldots, 6$, should be carefully chosen to obtain a critically damped response for the translational dynamics.

The stability of the translational and rotational dynamics in (61)-(63) and (70)-(72) is given next and is inspired from [20]. This stability analysis is carried out in a continuoustime framework. In practice the control algorithms have been implemented in a microcontroller using the fastest sampling rate.

The closed-loop system (61)-(63) and (70)-(72) can be represented as double integrators as follows

$$
\begin{aligned}
& \dot{x}_{1}=x_{2} \\
& \dot{x}_{2}=\tilde{u}
\end{aligned}
$$

The proposed control law has following structure

$$
\tilde{u}=-\sigma_{p}\left(\bar{k}_{1} x_{1}+\bar{k}_{2} x_{2}\right)
$$

then the candidate Lyapunov function is

$$
V\left(x_{1}, x_{2}\right)=\int_{0}^{\bar{k}_{1} x_{1}+\bar{k}_{2} x_{2}} \sigma_{p}(t) d t+\frac{1}{2} \bar{k}_{1} x_{2}^{2}
$$

for any constants $\bar{k}_{1}>0$ and $\bar{k}_{2}>0$ then

$$
\begin{aligned}
\dot{V}\left(x_{1}, x_{2}\right)= & \sigma_{p}\left(\bar{k}_{1} x_{1}+\bar{k}_{2} x_{2}\right)\left(\bar{k}_{1} \dot{x}_{1}+\bar{k}_{2} \dot{x}_{2}\right) \\
& +\bar{k}_{1} x_{2} \dot{x}_{2} \\
= & -\bar{k}_{2} \sigma_{p}^{2}\left(\bar{k}_{1} x_{1}+\bar{k}_{2} x_{2}\right)
\end{aligned}
$$

note that $V\left(x_{1}, x_{2}\right)$ is positive definite and $\dot{V}\left(x_{1}, x_{2}\right)$ is negative definite, therefore the closed-loop system is asymptotically stable.

\section{EXPERIMENTAL RESULTS}

This section presents the real-time experimental results to validate the performance of the rotorcraft during autonomous hover flight. The control gains of equations (61)-(63) and (70)-(72) were adjusted in practice to obtain an acceptable system's response, i.e. they were selected to obtain a fast aircraft response but avoiding mechanical oscillations as much as possible, they are shown in table I. The saturation values used in the attitude and horizontal control law are shown in table II. These parameters were also chosen in such a way that the aircraft attitude remains very close to a desired point.

The control law presented in section VI assumes that the rotational and translational dynamics were completely decoupled but in practice this does not exactly happens due to unsymmetrical rotor structures. To compensate these modeling errors we have trimmed the gains in the radio-control before the experiments.

\begin{tabular}{|c|c|c|c|c|c|}
\hline & Parameter & Value & & Parameter & Value \\
\hline$\psi$ & $a_{1}$ & 3.2 & $\mathrm{x}$ & $b_{1}$ & 2.3 \\
& $a_{2}$ & 1.5 & & $b_{2}$ & 1.2 \\
\hline$\theta$ & $a_{3}$ & 2.0 & $\mathrm{y}$ & $b_{3}$ & 2.3 \\
& $a_{4}$ & 0.3 & & $b_{4}$ & 1.2 \\
\hline$\phi$ & $a_{5}$ & 2.0 & $\mathrm{z}$ & $b_{5}$ & 3.1 \\
& $a_{6}$ & 0.3 & & $b_{6}$ & 1.8 \\
\hline
\end{tabular}

TABLE I

CONTROLLER PARAMETERS VALUES

\begin{tabular}{|l|l|l|}
\hline & Parameter & Saturation Value \\
\hline Attitude control & $\sigma_{a}$ & 150 \\
\hline Horizontal control & $\sigma_{b}$ & 250 \\
\hline
\end{tabular}

TABLE II

SATURATION PARAMETERS VALUES

The scene used to develop this experiment is composed of a red rectangle that contains inside a smaller white rectangle. The couple of rectangles have the same orientation, i.e. their edges are parallels to each other, moreover they have the same gravity center. This target is placed on a white background and the dimension is well known. With this target configuration the keypoints extraction is made easier, because the keypoints are the vertices of rectangles. Then we have up to 8 points to work.

The initial position is taken as desired position in the $x-$ $y$ plane. It set to $x_{d}=0 \mathrm{~cm}, y_{d}=140 \mathrm{~cm}$ and $z=60$ $\mathrm{cm}$. The time elapsed between two consecutive frames acquired is around $55 \mathrm{~ms}$, which means that the image sample rate is around 18 FPS.

In the experiment, the rotary flying machine was stabilized in hover flight applying the proposed control law and using the measures and estimations from the IMU and stereo vision system. The desired values to attitude angles to develop a hover flight is zero. As is shown in Figure 7 the control strategy for stabilizing the attitude performed well because the angles are very close to the origin. The estimated linear position in $x-y$ plane obtained from the stereo vision system is plotted in Figure 8. While the translational speeds are shown in Figure 9. For security reasons the eight rotor rotorcraft altitude is controlled in open loop using the radio transmitter. Figure 10 shows the control signals applied to front and left lateral motors. 

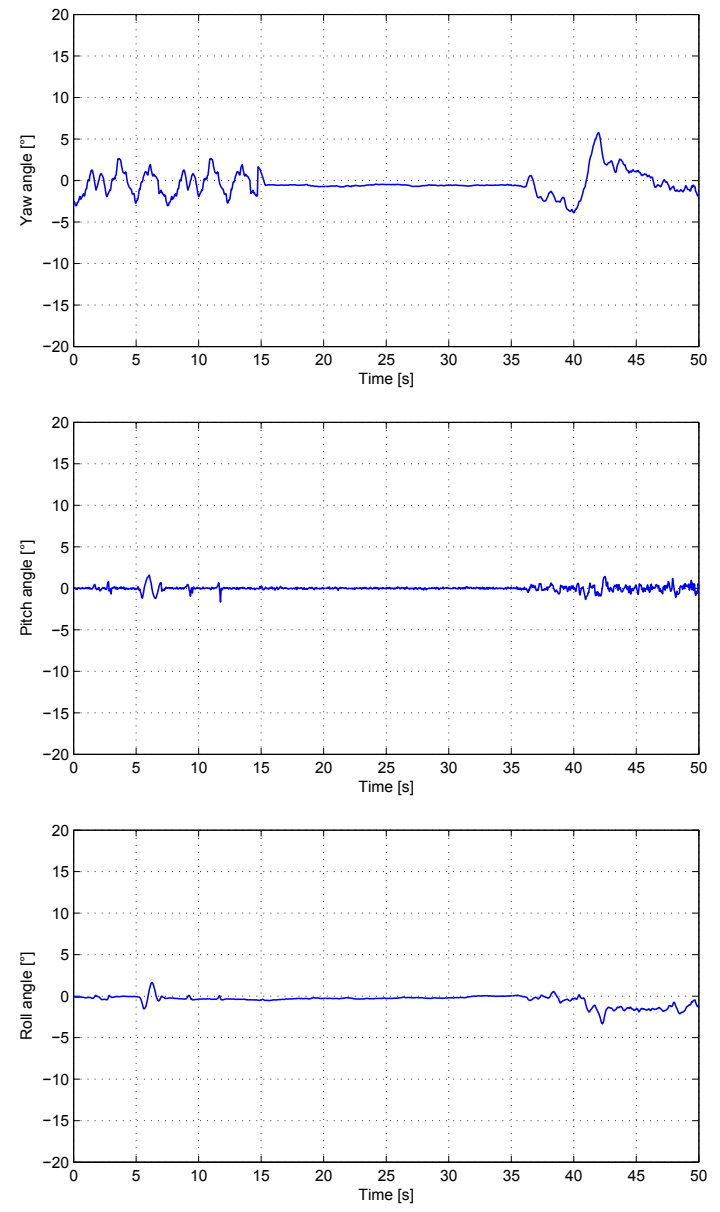

Fig. 7. Attitude behavior of eight-rotor rotorcraft.
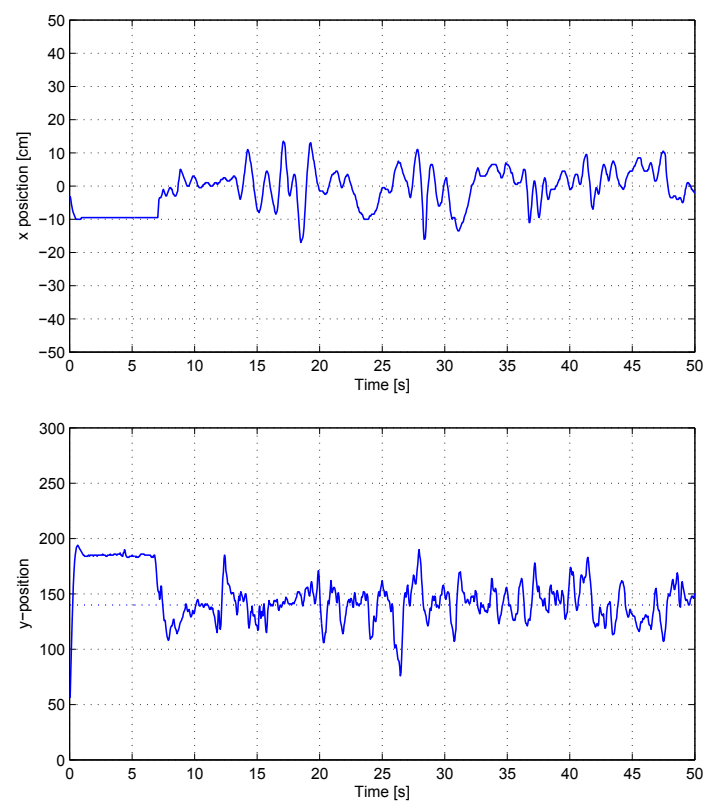

Fig. 8. Position behavior of eight-rotor rotorcraft using stereo vision.
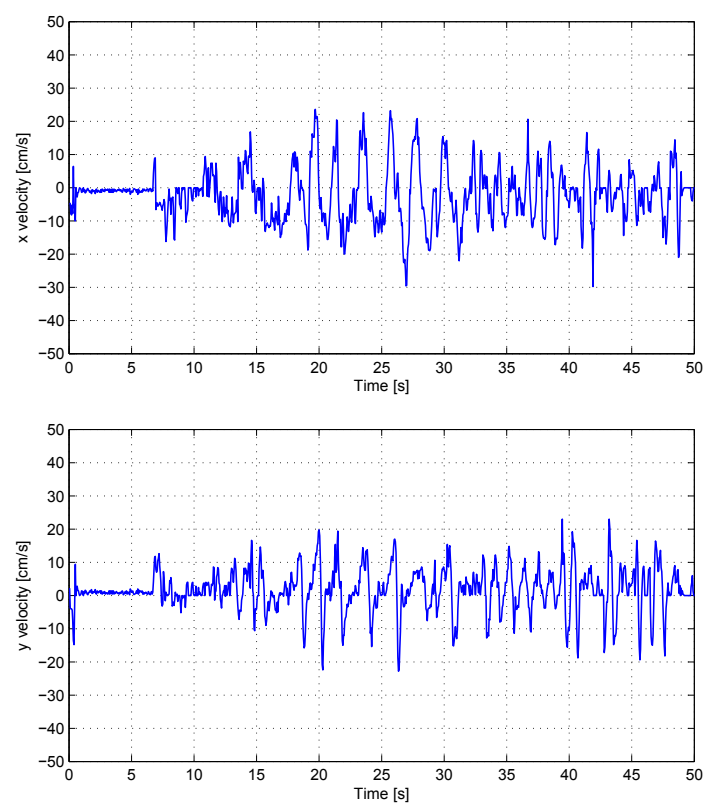

Fig. 9. Velocity behavior of eight-rotor rotorcraft using stereo vision.
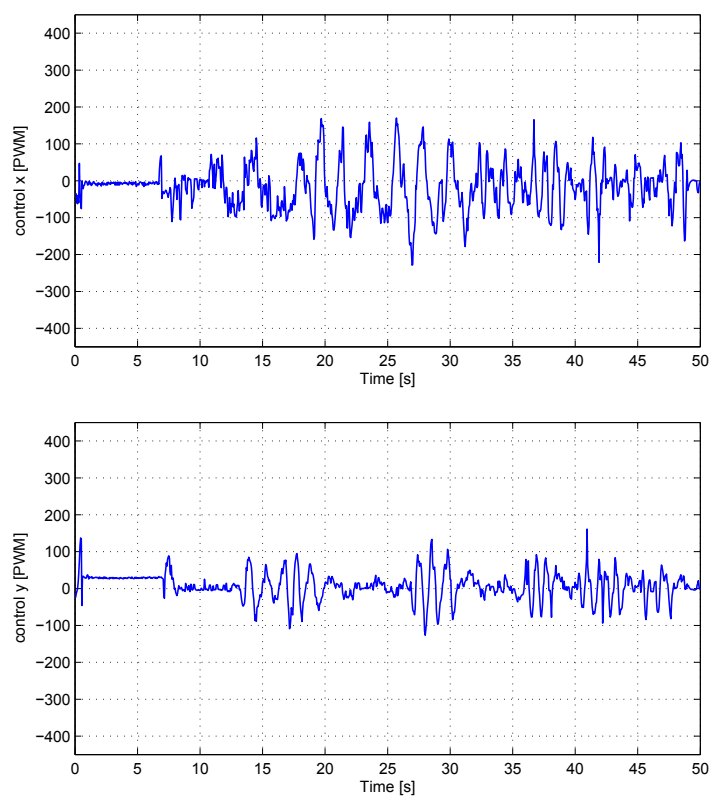

Fig. 10. Control signal applied to lateral motors. Top, control signal for lateral front motor. Bottom, control signal for lateral left motor. 


\section{CONCLUSIONS}

This paper presents a navigation system based on a semi embedded stereo vision system together with an IMU. This combination of measurement strategies has many advantages because one works very well at low speeds (vision system) and the other at high speeds (inertial sensors). On one hand the visual system measurements are used to control the translational dynamics, on the other hand the IMU is used to control the rotational dynamics. Both work at different sample rate.

Due to mechanical and aerodynamic features of the mini helicopter, the translational and rotational dynamics are almost decoupled each other. Taking advantage of this property we have obtained a simplified dynamical model of the rotorcraft. This model is given by six independent double integrators which have been stabilized using sature proportional-derivative (sPD) control. The real-time experiments have shown an acceptable performance of the flying machine applying the control law and sensing system proposed.

\section{REFERENCES}

[1] P. Castillo, R. Lozano and A. Dzul, "Modelling and Control of MiniFlying Machines", Springer-Verlag in Advances in Industrial Control, July 2005. ISBN: 1-85233-957-8

[2] Etkin B., "Dynamics of Flight", John Wiley and Sons, Inc., New York, 1959.

[3] O. Faugeras, B. Hotz, H. Mathieu, T. Viville, Z. Zhang, P. Fua, E. Thron, L. Moll, G. Berry, J. Vuillemin, P. Bertin et C. Proy, "Real time correlation based stereo: algorithms, implementations and applications", Technical Report 2013, INRIA, 1993.

[4] H. Goldstein, "Classical Mechanics", Addison Wesley Series in Physics, Adison-Wesley, U.S.A., second edition, 1980.

[5] R. Hartley et A. Zisserman, "Multiple View Geometry in Computer Vision", Cambridge University Press, 2nd. Edition, 2004.

[6] Z. He, R.V. Iyer et P. Chandler, "Vision-based UAV Flight Control and Obstacle Avoidance", Proc. IEEE American Control Conference, pp. 2166-2170, 2006

[7] S. Hrabar and G.S. Sukhatme, P. Corke, K. Usher et J. Roberts, "Combined Optic-Flow and Stereo-Based Navigation of Urban Canyons for a UAV', IEEE International Conference on Intelligent Robots and Systems IROS 2005 .

[8] Wang, L.K. Hsieh, S.-C. Hsueh, E.C.-W. Fei-Bin Hsaio, Kou-Yuan Huang. "Complete pose determination for low altitude unmanned aerial vehicle using stereo vision",Intelligent Robots and Systems, 2005. (IROS 2005).

[9] Cory S. Sharp, Omid Shakernia, S. Shankar Sastry. "A Vision System for Landing an Unmanned Aerial Vehicle", 2001 IEEE International Conference on Robotics and Automation.

[10] Seunghyun Leen, Seungho Yoon, Hyoun Jin Kim, Youdan Kim. "Wireless stereo vision system development for rotary-wing UAV guidance and control", 3rd International Conference on Sensing Technology, 2008.

[11] Demonceaux, C. Vasseur, P. Regard, C. "Omnidirectional vision on UAV for attitude computation", Proceedings 2006 IEEE International Conference on Robotics and Automation, 2006. ICRA 2006.

[12] B. W. McCormick Jr., “ Aerodynamics of VSTOL Flight”, Dover Publication Inc., 1999.

[13] S. Merhav, "Aerospace sensors systems and applications", SpringerVerlag, 1996.

[14] R. Horaud and O. Monga "Vision pqr ordinqteur: Outils fundamentaux", Hermes 2nd. Edition, 2003.

[15] H. Romero, R. Benosman and R. Lozano, "Stabilization and location of a four rotors helicopter applying vision". In Proc. American Control Conference ACC. pp. 3931-3936, 2006.

[16] H. Romero, "Modlisation et asservissement visuel d'un mini hlicoptre". Ph.D. Thesis, UTC HEUDIASyC Compigne France, 2008.

[17] J.Z. Sasiadek and P. Hartana, "Sensor Fusion for Navigation of an Autonomous Unmanned Aerial Vehicle. In Proc. International Conference on Robotics and Automation, Vol.4 pp. 429-434, 2004.
[18] S. Salazar, H. Romero, R. Lozano and P. Castillo, "Real-Time stabilization of an eight rotors UAV using optical flow, IEEE Transaction on Robotics, Vol. 25 No. 5, 2009.

[19] P. Sturm, "Vision 3D non calibre: reconstruction projective et tude des mouvements critiques pour l'auto-calibrage", Thse de doctorat, Institute National Polytechnique de Grenoble.

[20] Sussman H and Yang Y., " the OnStabilizability of Multiple Integrators by Means of Bounded Feedback controls" In Proceedings of the 30th IEEE Conference on Decision and Control, Brighton, UK, Dec. 1991., IEEE Publications, New York, 1991, pp. 70-72. 1991.

[21] A.D. Wu, E.N. Johnson et A.A. Proctor, "Vision-Aided Inertial Navigation for Flight Control", AIAA Journal of Aerospace Computing, Information, and Communication, Vol. 2, No. 9, pp. 348-360, September 2005.

[22] Z. Zhang , A Flexible New Technique for Caméra Calibration, IEEE Transactions on Pattern Analysis and Machine Intelligence, Vol. 22 , pp 1330-1334. 